\title{
Measurable Lattice Effects on the Charge and Magnetic Response in Graphene
}

\author{
G. Gómez-Santos and T. Stauber \\ Departamento de Física de la Materia Condensada and Instituto Nicolás Cabrera, Universidad Autónoma de Madrid, \\ E-28049 Madrid, Spain
}

(Received 11 November 2010; published 27 January 2011)

\begin{abstract}
The simplest tight-binding model is used to study lattice effects on two properties of doped graphene: (i) magnetic orbital susceptibility and (ii) regular Friedel oscillations, both suppressed in the usual Dirac cone approximation. (i) An exact expression for the tight-binding magnetic susceptibility is obtained, leading to orbital paramagnetism in graphene for a wide range of doping levels which is relevant when compared with other contributions. (ii) Friedel oscillations in the coarse-grained charge response are considered numerically and analytically and an explicit expression for the response to lowest order in lattice effects is presented, showing the restoration of regular $2 d$ behavior, but with strong sixfold anisotropy.
\end{abstract}

PACS numbers: 81.05.ue, 73.22.Pr, 75.20.-g, 75.70.Ak

Introduction.-The recent experimental realization [1] of graphene, the single layer honeycomb lattice of carbon atoms that forms graphite, has unleashed an explosion of activity. High expectations have been put on profiting from its peculiar electronic, mechanical, optical (and perhaps magnetic) properties, when tailored at the nanoscale [2]. The existence of linearly dispersing bands around two nodal points (massless Dirac fermions with velocity $v \sim 10^{6} \mathrm{~m} / \mathrm{s}$ ) form the basis of graphene's most notable electronic properties [3].

Many theoretical studies of graphene are done within scaling limit or Dirac cone approximation, that is, assuming strictly linear energy dispersion around the nodal points. Although this approach is successful in explaining many experimental facts, it has limitations too. Obvious examples are provided by magnitudes for which the Dirac cone approximation provides a null result. In this Letter we are concerned with two such magnitudes: (i) the magnetic susceptibility and (ii) regular Friedel oscillations, both rendered zero at finite doping in the scaling limit.

(i) Strong and peculiar diamagnetism characterizes graphene, as first discussed by McClure to explain graphite, nature's best diamagnet. He found that, for the twodimensional Dirac model, the diamagnetic susceptibility was given by a delta function of the chemical potential [4]. This result implies that there is no magnetic response when the chemical potential is shifted from the neutrality point. This is in clear contrast with recent experimental findings of paramagnetism in graphene [5].

Here we will show that lattice effects, neglected in the scaling limit, render finite and sizable the magnetic response. We employ the formalism of Fukuyama [6], whose original formula was first applied to graphite [7] and subsequently to graphite intercalated compounds [8,9], which is here extended by an additional term required to provide the exact susceptibility for a general tight-binding model. The magnetic response for arbitrary chemical potential is obtained, finding orbital paramagnetism (OP) over a wide range of fillings. Its value close to the neutrality point is compared with other sources (core diamagnetism, spin paramagnetism, and interaction's induced orbital paramagnetism) and shown to be a relevant contribution.

(ii) Graphene's charge response around localized perturbations is also peculiar [10-12]. While ordinary $2 d$ systems show the familiar Friedel $2 k_{F}$ oscillations decaying as $1 / r^{2}$, graphene's coarse-grained response in the scaling limit does so but with an additional power. Graphene's lack of regular $2 d$ Friedel oscillations is linked to isospin (or chiral) conservation and thus provides the possibility of direct observation of the nature of graphene's excitations [13].

Here we show, numerically and analytically, that lattice effects restore the standard $2 d$ behavior. An explicit expression for the charge response is obtained to lowest order in lattice effects, exhibiting the usual $1 / r^{2}$ decay and a pronounced sixfold anisotropy, with maxima along the bond's directions.

One might ask why we treat two at first sight such distinct topics on the same footing. The reason is that within the Dirac cone approximation the static transverse current-current as well as the charge-charge correlation function yield $\chi(q)=a+b q^{2}+\ldots$ with $b \sim \delta\left(E_{F}\right)$ and $E_{F}$ the Fermi level. Lattice contributions to the response, given by $\chi^{\text {lattice }} \sim q^{2}$ for finite filling factor, are thus suppressed in the same peculiar way.

Tight-binding model and Dirac cone approximation.We describe graphene by the simplest tight-binding Hamiltonian $H=-t \sum_{\boldsymbol{R}, \boldsymbol{\delta}} a_{\boldsymbol{R}}^{\dagger} b_{\boldsymbol{R}+\boldsymbol{\delta}}+$ H.c. with hopping amplitude $-t$ between nearest neighbor atoms in $A$ and $B$ sublattices joined by vectors $\boldsymbol{\delta}_{1}=(0, a)$ and its $\pm 120^{\circ}$-rotated versions $\boldsymbol{\delta}_{2,3}$. The spectrum is $E^{ \pm}(\boldsymbol{k})=$ $\pm|t S(\boldsymbol{k})|$, where $S(\boldsymbol{k})=\sum_{i} \exp \left(i \boldsymbol{k} \cdot \boldsymbol{\delta}_{i}\right)$. It develops a well-known linear dispersion $E^{ \pm}(\boldsymbol{k})= \pm \hbar v k^{\prime}$ with $\boldsymbol{k}^{\prime}=$ $\boldsymbol{k}-\boldsymbol{K}_{1,2}$ around two points in the Brillouin zone, $\boldsymbol{K}_{1,2}=$ $\pm\left(\frac{4 \pi}{3 \sqrt{3} a}, 0\right)$. 
For the orbital magnetic response, the Dirac cone approximation leads to a diamagnetic susceptibility depending on Fermi level $E_{F}$ as [4]

$$
\chi_{\text {orb }}^{\text {Dirac }}=-\mu_{0} \frac{g_{s} g_{v}}{6 \pi} e^{2} v^{2} \delta\left(E_{F}\right),
$$

with vacuum permeability $\mu_{0}$ (SI units), spin and valley degeneracies $g_{s}=g_{v}=2$, and unit charge $e$. The peculiar relation, known to be at the basis of graphite's large diamagnetism [7], implies that doped graphene shows no magnetic orbital response at finite doping.

Also the charge response to a local impurity $V=u \delta(\boldsymbol{r})$ shows peculiar behavior since the first $q$ derivative of its susceptibility is continuous at $q=2 k_{F}$. This results in an anomalous decay of the Friedel oscillations which in terms of the electronic carrier density $\rho_{e}$ reads [10]

$$
\frac{\delta \rho(r)}{\rho_{e}}=\frac{u \rho_{e}}{E_{F}} \frac{\cos \left(2 k_{F} r\right)}{\left(2 k_{F} r\right)^{3}}, \quad k_{F} r \gg 1 .
$$

In what follows, we will show that both results are substantially altered when lattice effects are included.

Magnetic response.-Fukuyama [6] has provided a convenient expression for the orbital magnetic susceptibility in noninteracting systems. The formula is exact only for a Hamiltonian of the canonical form $H=\frac{\boldsymbol{P}^{2}}{2 m}+V(\boldsymbol{R})$. Here we adapt Fukuyama's procedure to obtain the exact orbital response of a tight-binding Hamiltonian. Employing the current operator of the tight-binding model given in Ref. [14], linear response theory yields the following expression for the orbital susceptibility [15]:

$$
\begin{aligned}
\chi_{\mathrm{orb}}= & -\mu_{0} \frac{e^{2}}{\hbar^{2}} \frac{g_{s}}{2 \pi} \operatorname{Im} \int d E n_{F}(E) \\
& \times \frac{1}{A} \sum_{k} \operatorname{Tr}\left\{\hat{\gamma}^{x} \hat{\mathcal{G}} \hat{\gamma}^{y} \hat{\mathcal{G}} \hat{\gamma}^{x} \hat{\mathcal{G}} \hat{\gamma}^{y} \hat{\mathcal{G}}\right. \\
& \left.+\frac{1}{2}\left(\hat{\mathcal{G}} \hat{\gamma}^{x} \hat{\mathcal{G}} \hat{\gamma}^{y}+\hat{\mathcal{G}} \hat{\gamma}^{y} \hat{\mathcal{G}} \hat{\gamma}^{x}\right) \hat{\mathcal{G}} \frac{\partial \hat{\gamma}^{y}}{\partial k_{x}}\right\}
\end{aligned}
$$

with the $2 \times 2$ matrix $\hat{G}_{k}(E)=\left(E+i 0^{+}-\hat{H}_{k}\right)^{-1}, \hat{\gamma}_{k}=$ $\nabla_{\boldsymbol{k}} \hat{H}_{\boldsymbol{k}}$, and $\hat{H}_{\boldsymbol{k}}=-\operatorname{Re}[t S(\boldsymbol{k})] \sigma_{x}+\operatorname{Im}[t S(\boldsymbol{k})] \sigma_{y}$.

This expression for $\chi_{\text {orb }}$ transcends the model of the initial Hamiltonian, and turns out to be correct for any tight-binding system. Equation (3) coincides with Fukuyama's original result [6] except for the second term. The difference stems from the standard isotropic $\boldsymbol{P}$ dependence in Fukuyama's $H=\frac{\boldsymbol{P}^{2}}{2 m}+V(\boldsymbol{R})$, where one has $\frac{\partial \hat{\gamma}^{y}}{\partial k_{x}}=0$. Such cancellation does not apply in a generic tight-binding case.

The above formula has been applied to numerically calculate the orbital susceptibility in graphene as a function of Fermi level $E_{F}$. The most prominent feature is, of course, the delta function at the band center of Eq. (1), which comes with the known analytical value. We can thus extract the lattice contribution from the numerical results by writing

$$
\chi_{\mathrm{orb}}=-\mu_{0} \frac{g_{s} g_{v}}{6 \pi} e^{2} v^{2} \delta\left(E_{F}\right)+\Delta \chi_{\mathrm{orb}}
$$

The calculated lattice contribution $\Delta \chi_{\text {orb }}$ is plotted in Fig. 1 in units of $\chi_{0}=\mu_{0} \hbar^{-2} e^{2}|t| a^{2}$. The lattice origin of $\Delta \chi_{\text {orb }}$ becomes evident if one artificially sets $a \rightarrow 0$ and $t \rightarrow \infty$ while $a t \propto v=$ const (scaling limit): then $\Delta \chi_{\text {orb }} \rightarrow 0$, leaving the Dirac cone result [Eq. (1)] as the sole response.

Discussion.-The orbital response is usually associated with diamagnetism, so a noteworthy aspect of the lattice contribution in Fig. 1 is its paramagnetic character over most of the band, even diverging at the van Hove points [16]. From Eq. (3), one finds the following sum rule: $\int d E_{F} \chi_{\text {orb }}\left(E_{F}\right)=0$. The existence of OP is thus a necessary consequence to cancel the large diamagnetic contribution of the scaling limit at the band center, see Eq. (4). Only at the band edges Landau diamagnetism emerges, as expected.

Now, we compare lattice's OP with other contributions in the region $E_{F} \sim 0$, relevant for gate-doped graphene. Within our noninteracting model, the only remaining magnetic contribution is Pauli's spin paramagnetism, given by $\chi_{\text {spin }} / \mu_{0}=2 \mu_{B}^{2} \rho\left(E_{F}\right)$, where $\mu_{B}$ is the Bohr magneton and $\rho\left(E_{F}\right)$ is the density of states (per spin). This spin contribution is plotted in Fig. 1, where it is seen that it cannot compete with the dominant orbital contribution for low carrier densities $\rho_{e}$.

Core electrons, not considered in our $\mathrm{pp} \pi$ band Hamiltonian, are another source of (dia)magnetic response. The estimate of Ref. [17], $\chi_{\text {core }} \sim-4.8 \times 10^{-6} \mathrm{emu} / \mathrm{mol}$, translates into $\chi_{\text {core }} \sim-0.13 \chi_{0}$, a value marked with an arrow in the scale of Fig. 1. Again, the orbital contribution for low $\rho_{e}$ is comparable or greater than this estimate of core diamagnetism.

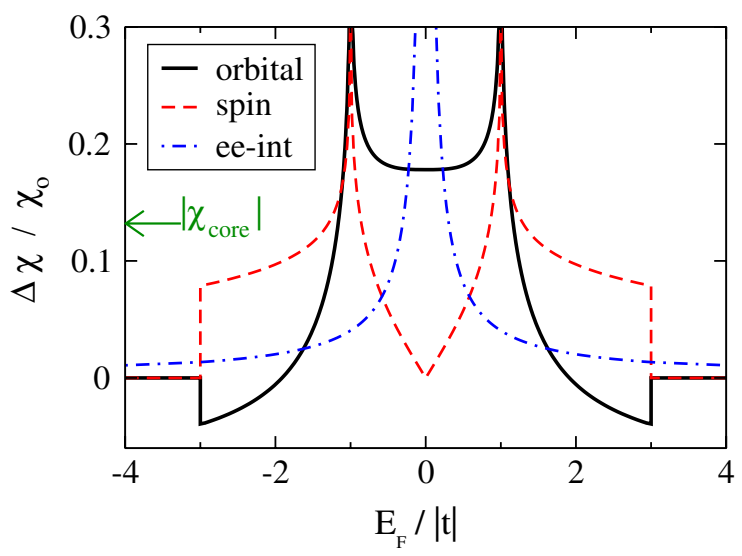

FIG. 1 (color online). Continuous line: lattice contribution to the orbital magnetic susceptibility $\left(\Delta \chi_{\mathrm{orb}}\right)$ in units of $\chi_{0}=$ $\mu_{0} \hbar^{-2} e^{2}|t| a^{2}$. Dashed line: Pauli's spin paramagnetic contribution. Dash-dotted line: orbital magnetic susceptibility from Coulomb electron-electron interactions [Eq. (5)]. The arrow marks an estimate of the absolute value of the diamagnetic contribution from core electrons [17]. 
Recently, the effect of Thomas-Fermi screened Coulomb and contact interaction on the magnetic response was considered to first order within the Dirac cone approximation, both leading to OP at finite [18]. For the Coulomb case it reads

$$
\chi_{e e}=\chi_{0} \frac{C}{E_{F} /|t|},
$$

with an interaction dependent constant $C \sim 0.04$, suitable for graphene over $\mathrm{SiO}_{2} . \chi_{e e}$ is compared with the lattice contribution in Fig. 1. Graphene's poor screening causes the divergence of $\chi_{e e}$ when $E_{F} \rightarrow 0$, making this contribution dominant. But even in this unfavorable case, the lattice contribution is not negligible. For instance, $\Delta \chi_{\text {orb }} \gtrsim \chi_{e e}$ for doping levels $n \gtrsim 3.7 \times 10^{13} \mathrm{~cm}^{-2}$, and for a typical doping $n \sim 4 \times 10^{12} \mathrm{~cm}^{-2}$ one has $\chi_{e e} \sim 3 \Delta \chi_{\text {orb }}$.

Finally, if (by some external means) screening were truly effective so that interactions could be described by a contact term $v_{0}$, Ref. [18] provides the following expression for the interaction's promoted orbital paramagnetism:

$$
\chi_{e e}=g_{s} g_{v} \mu_{0} e^{2} \hbar^{-2} \frac{13}{256 \pi^{2}} v_{0} \sim 0.027 \chi_{0} \frac{U}{|t|},
$$

where we have written the interaction as $v_{0}=U A_{c} / 2$, with a Hubbard-like energy $U$, and area per unit cell $A_{c}=\frac{3 \sqrt{3} a^{2}}{2}$. In order to compare this contribution with that of the lattice, we calculate the size of $U$ required for $\chi_{e e}$ of Eq. (6) to match the lattice contribution around $E_{F} \sim 0$. The answer turns out to be $U \sim 7|t|$, a value substantially larger than current estimates for graphene. This implies that, in any reasonable scenario of contact interactions, the lattice orbital contribution to paramagnetism would be the key player in the magnetic response of doped graphene.

Let us close with a remark on the effect of next-nearest neighbor hopping, temperature, and disorder. As was already noted in Ref. [9], $t^{\prime} \approx 0.1 t$ leads to a considerable electron-hole asymmetry in the magnetic response; the above qualitative discussion on the relevance of the several contributions, though, is not altered. Temperature and disorder [19] broadens the diamagnetic delta peak, such that lattice effects gradually lose relevance at a given temperature or disorder when the chemical potential decreases to zero.

Charge response.-The linear, static charge response of graphene is given by

$$
\Pi(\boldsymbol{q})=\frac{1}{A} \sum_{\boldsymbol{k}_{1}, s, s^{\prime}= \pm} f_{s \cdot s^{\prime}}\left(\boldsymbol{k}_{1}, \boldsymbol{k}_{2}\right) \frac{n_{F}\left(E^{s}\left(\boldsymbol{k}_{1}\right)\right)-n_{F}\left(E^{s^{\prime}}\left(\boldsymbol{k}_{2}\right)\right)}{E^{s}\left(\boldsymbol{k}_{1}\right)-E^{s^{\prime}}\left(\boldsymbol{k}_{2}\right)},
$$

with $\boldsymbol{k}_{1}=\boldsymbol{k}_{2}+\boldsymbol{q}$, and the prefactor $f_{ \pm}$

$$
f_{ \pm}\left(\boldsymbol{k}_{1}, \boldsymbol{k}_{2}\right)=\frac{1}{2} \pm \frac{1}{2} \operatorname{Re}\left(\frac{S\left(\boldsymbol{k}_{1}\right)}{\left|S\left(\boldsymbol{k}_{1}\right)\right|} \frac{S^{*}\left(\boldsymbol{k}_{2}\right)}{\left|S\left(\boldsymbol{k}_{2}\right)\right|}\right) .
$$

Friedel oscillations are caused by intraband transitions [+ sign in Eq. (8)] with $q / 2 \sim k_{F}$, the Fermi wave vector (measured from the Dirac point). To understand graphene's peculiarity, let us set $f_{+}=1$ in Eq. (7), and call the associated Lindhard-like response $\tilde{\Pi}$. Then, the dominant singularity in $\tilde{\Pi}$, corresponding to transitions across the Fermi surface, leads to the prototypical $2 d$ square-root behavior

$$
\tilde{\Pi}(q) \sim \frac{g_{s} g_{v} \sqrt{k_{F}}}{2 \pi \hbar v} \sqrt{q-2 k_{F}} \Theta\left(q-2 k_{F}\right),
$$

where $\Theta$ is Heaviside's function and we have ignored any distortion of the isotropic Fermi surface around the two Dirac points. Within the Dirac cone approximation, the prefactor $f_{+}$in Eq. (8) crucially vanishes for states on opposite sides of the Fermi surface. The physical interpretation is well known [10]: the involved states have opposite chirality and cannot be coupled by a perturbation diagonal in sublattice index. Nevertheless, this exact cancellation of $f_{+}$holds true only in the scaling limit $k_{F} a \rightarrow 0$, and a finite value of $k_{F} a$ renders $f_{+}$finite, something we generically label as lattice effect.

To see if this square-root behavior is present also for the true prefactor $f_{+}$as given in Eq. (7), we numerically analyze the response derivatives which we conveniently write as

$$
\begin{aligned}
\frac{\partial \Pi(\boldsymbol{q})}{\partial q_{\alpha}}= & -\frac{g_{s}}{A 2 \pi} \operatorname{Im} \int d E n_{F}(E) \sum_{\boldsymbol{k}} \operatorname{Tr}\left\{\hat{\boldsymbol{G}}_{\boldsymbol{k}}(E) \hat{\gamma}_{\boldsymbol{k}}^{\alpha}\right. \\
& \left.\times \hat{\boldsymbol{G}}_{\boldsymbol{k}}(E)\left(\hat{\boldsymbol{G}}_{\boldsymbol{k}-\boldsymbol{q}}(E)-\hat{\boldsymbol{G}}_{\boldsymbol{k}+\boldsymbol{q}}(E)\right)\right\} .
\end{aligned}
$$

We observe a clear anisotropy with a pronounced spike that hints at a singular behavior for the results in the $y$ direction, absent in the $x$ direction, where the behavior is closer to that of the (analytically known) Dirac cone approximation [15]. The numerical results strongly suggest the restoration of a regular $2 d$ response but with strong anisotropy. This is confirmed by the analytical treatment that follows.

Now we obtain analytically the charge response to lowest order in lattice effects. We start with the determination of the prefactor $f_{+}(\boldsymbol{q})$, that is, Eq. (8) for two states on opposite sides of the Fermi surface: $\boldsymbol{k}_{2}$ and $\boldsymbol{k}_{1}=\boldsymbol{k}_{2}+\boldsymbol{q}$, such that the vector $\boldsymbol{q}$ corresponds to the square-root singularity in the response. The latter condition requiring the $\boldsymbol{q}$-linked portions of the Fermi surface to be parallel. Upon a Jacobi-Anger expansion of the terms $\exp \left(i \boldsymbol{k} \cdot \boldsymbol{\delta}_{i}\right)$, the structure factor can be written as $S(\boldsymbol{k})=3 \sum_{n} J_{-1+3 n}\left(k^{\prime} a\right) e^{i(-1+3 n) \phi}$ where $J_{n}$ are Bessel functions of the first kind, and the separation from the Dirac point is $\boldsymbol{k}^{\prime}=\boldsymbol{k}-\boldsymbol{K}_{1}$, with polar coordinates $\left(k^{\prime}\right.$, $\phi)$. To lowest order in lattice effects, only $J_{-1}=-J_{1}$ and $J_{2}$ are to be retained. Then, the requirement $|S(\boldsymbol{k})|=$ const leads to the following expression for the Fermi surface in polar coordinates: 


$$
k_{F}^{\prime}(\phi)=k_{F}\left(1+\frac{k_{F} a}{4} \cos (3 \phi)+\mathcal{O}\left(k_{F} a\right)^{2}\right),
$$

where we have parametrized the Fermi energy by the wouldbe Fermi wave vector in the isotropic limit: $E_{F}=\hbar v k_{F}$.

To lowest order, the condition of parallel pieces of Fermi surface leads to the following relation between polar angles $\left(\phi_{1,2}\right)$ of the involved $\boldsymbol{k}$ points: $\phi_{1}=\phi_{q}-\delta \phi$ and $\phi_{2}=$ $\phi_{q}+\pi+\delta \phi$ with the lattice correction $\delta \phi=\frac{3}{4}\left(k_{F} a\right) \times$ $\sin \left(3 \phi_{q}\right)$ and $\phi_{q}$ the polar angle of the joining vector $\boldsymbol{q}$ with modulus $q=2 k_{F}\left(1+\mathcal{O}\left(k_{F} a\right)^{2}\right)$. We can now write the phase of $S\left(\boldsymbol{k}^{\prime}\right)$ as

$$
\frac{S\left(\boldsymbol{k}^{\prime}\right)}{\left|S\left(\boldsymbol{k}^{\prime}\right)\right|} \sim-e^{-i(\phi+\theta)},
$$

where $\phi$ is the polar angle of $\boldsymbol{k}^{\prime}$ and $\theta$ is the lattice correction to that phase given to lowest order by $\theta=\frac{1}{4} \times$ $\left(k_{F} a\right) \sin (3 \phi)$. This leads to the final expression for the prefactor

$$
f_{+}(\boldsymbol{q})=\frac{\left(k_{F} a\right)^{2}}{8}\left(1-\cos \left(6 \phi_{q}\right)\right)+\mathcal{O}\left(k_{F} a\right)^{3},
$$

where $\left(2 k_{F}, \phi_{q}\right)$ are the polar coordinates of $\boldsymbol{q}$ and which holds for both Dirac points. We note that the result of Eq. (13), although the lowest finite order in a $k_{F}$ expansion, already represents an excellent approximation for sizable Fermi levels well within the range of gate-voltage doped graphene's samples [15].

Combining Eqs. (9) and (13), the dominant singularity of the true response is

$$
\begin{aligned}
\Pi \sim & \frac{\left(k_{F} a\right)^{2}}{8}\left(1-\cos \left(6 \phi_{q}\right)\right) \frac{g_{s} g_{v} \sqrt{k_{F}}}{2 \pi \hbar v} \\
& \times \sqrt{q-2 k_{F}} \Theta\left(q-2 k_{F}\right) .
\end{aligned}
$$

We can now determine the density response associated to a local perturbing potential $V=u \delta(\boldsymbol{r})$ given by $\frac{\delta \rho(\boldsymbol{r})}{u}=$ $\frac{1}{(2 \pi)^{2}} \int d^{2} q e^{i q \cdot r} \Pi(\boldsymbol{q})$. The remaining integral is obtained from standard techniques [20], leading to the following asymptotic behavior for Friedel oscillations:

$$
\frac{\delta \rho(\boldsymbol{r})}{\rho_{e}}=-\frac{\left(k_{F} a\right)^{2}}{2 \sqrt{2}}\left(1-\cos \left(6 \phi_{r}\right)\right) \frac{u \rho_{e}}{E_{F}} \frac{\sin \left(2 k_{F} r\right)}{\left(2 k_{F} r\right)^{2}} .
$$

While its $r^{-2}$ behavior is standard for a $2 d$ system, its true origin as a lattice contribution to an otherwise null result (to order $r^{-2}$ ) is revealed by its amplitude, vanishing as $\left(k_{F} a\right)^{2}$ in the scaling limit $a \rightarrow 0$, and by its anisotropy reflecting the sixfold symmetry of the lattice.

Comparing Eq. (15) with the result coming from the Dirac cone approximation, Eq. (2), we first notice the phase shift of $\pi / 2$. We further find for the crossover length between anomalous and regular Friedel oscillations $r_{c} \sim$ $k_{F}^{-1}\left(k_{F} a\right)^{-2}$. For $k_{F}=1 \mathrm{~nm}^{-1}$, we have $r_{c} \sim 100 \mathrm{~nm}$ which corresponds to an impurity concentration of $n_{i} \sim 10^{10} \mathrm{~cm}^{-2}$, recently found to be the intrinsic concentration of local impurities in graphene [21]. We thus expect Friedel oscillations to show anisotropic behavior and modify the RKKY interactions for doping levels $E_{F} \gtrsim 0.5 \mathrm{eV}$.

Summary.-The simplest tight-binding model has been employed to study the lattice contribution to the magnetic susceptibility and (coarse-grained) charge response of doped graphene, for which the Dirac cone approximation produces a null result. The lattice magnetic response shows orbital paramagnetism for a wide range of filling factors, representing a relevant contribution when compared to other sources such as core diamagnetism, spin paramagnetism, and electron-electron interaction induced orbital paramagnetism. Lattice effects restore the $2 d$ regular behavior for the coarse-grained charge response, with Friedel oscillations decaying as $r^{-2}$ but with pronounced sixfold anisotropy, with maxima along bond's directions.

We are grateful to F. Guinea for useful discussions. This work has been supported by FCT under Grant No. PTDC/ FIS/101434/2008 and MIC under Grant No. FIS201021883-C02-02.

[1] K. S. Novoselov et al., Science 306, 666 (2004).

[2] A. K. Geim, Science 324, 1530 (2009).

[3] A. H. C. Neto et al., Rev. Mod. Phys. 81, 109 (2009).

[4] J. W. McClure, Phys. Rev. 104, 666 (1956); M. Koshino, Y. Arimura, and T. Ando, Phys. Rev. Lett. 102, 177203 (2009); A. Principi, M. Polini, and G. Vignale, Phys. Rev. B 80, 075418 (2009).

[5] M. Sepioni et al., Phys. Rev. Lett. 105, 207205 (2010).

[6] H. Fukuyama, Prog. Theor. Phys. 45, 704 (1971).

[7] M.P. Sharma, L. G. Johnson, and J.W. McClure, Phys. Rev. B 9, 2467 (1974).

[8] S. A. Safran and F. J. DiSalvo, Phys. Rev. B 20, 4889 (1979).

[9] J. Blinowski and C. Rigaux, J. Phys. (Paris) 45, 545 (1984).

[10] V. V. Cheianov and V.I. Fal'ko, Phys. Rev. Lett. 97, 226801 (2006).

[11] B. Wunsch et al., New J. Phys. 8, 318 (2006).

[12] E. Mariani et al., Phys. Rev. B 76, 165402 (2007); L. Brey, H. A. Fertig, and S. Das Sarma, Phys. Rev. Lett. 99, 116802 (2007); C. Bena, ibid. 100, 076601 (2008).

[13] I. Brihuega et al., Phys. Rev. Lett. 101, 206802 (2008).

[14] T. Stauber and G. Gómez-Santos, Phys. Rev. B 82, 155412 (2010).

[15] See supplemental material at http://ink.aps.org/ supplemental/10.1103/PhysRevLett.106.045504 for more details.

[16] G. Vignale, Phys. Rev. Lett. 67, 358 (1991).

[17] F. J. DiSalvo et al., Phys. Rev. B 20, 4883 (1979).

[18] A. Principi et al., Phys. Rev. Lett. 104, 225503 (2010).

[19] M. Koshino and T. Ando, Phys. Rev. B 75, 235333 (2007); M. Nakamura, ibid. 76, 113301 (2007).

[20] M. J. Lighthill, An Introduction to Fourier Analysis and Generalized Functions (Cambridge University Press, Cambridge, 1958).

[21] Z. H. Ni et al., Nano Lett. 10, 3868 (2010). 